\title{
Effect of Different Modulation Techniques Comparison of Linear MIMO Receivers
}

\author{
Saifullah Adnan \\ College of Information and \\ Communication Engineering, \\ Harbin Engineering University, \\ China.
}

\author{
Naveed Ur Rehman \\ College of Information and \\ Communication Engineering, \\ Harbin Engineering University, \\ China.
}

\author{
Mohammad Irshad \\ Zahoor \\ College of Information and \\ Communication Engineering, \\ Harbin Engineering University, \\ China.
}

\begin{abstract}
In wireless communication, Multiple-Input Multiple-Output (MIMO) is the most propitious technology that enhances the system capacity and data rate by using multiple antennas at the receiver and at the transmitter. Efficient signal detection at the receiver is complex task in MIMO systems. The computational complexity of equalization based linear detection algorithms such as Minimum Mean Square Error (MMSE) and Zero Forcing (ZF) is less than that of algorithmic schemes. The simulated results presented in this paper are using various MIMO system configurations from $2 \times 2$ to $8 \times 8$. The results are compared and analyzed on the basis of Bit Error Rate (BER) for M-QAM modulation techniques using MMSE, ZF reduced complexity algorithms under Rayleigh fading channel. Simulations results reveal that the MMSE detection offers better performance over ZF detection however for 64-QAM modulation both linear detection shows same performance.
\end{abstract}

\section{Keywords}

MIMO, ZF, MMSE, BER, M-QAM Modulation.

\section{INTRODUCTION}

MIMO is the antenna technology for radio communication that is used in transmitter and receiver hardware. For making the communication robust diversity modes are used with varying channels. The use of multiple antennas at the transmitter or the receiver end is required for the spatial diversity. MIMO systems can also be used to increase the data rate instead of improving robustness [1]. The use of multiple antennas at the receiver and transmitter takes the advantage of multipath by which better throughput and bit error improvement is obtained. Spatial multiplexing is advantageous as it provides significant increase in transmission data rate of the wireless channel in exploiting the random fading and multi-path effects [2]. Future generation wireless systems main goal is highperformance, high data-rate, and optimum utilization of the bandwidth that is the MIMO systems by which that goal is achieved [3]

In MIMO systems each symbol is transmitted from the transmitter simultaneously, the received symbols at the receiver are added. As a result the receiver separate each symbol according to the symbols transmitted. Digital modulation is more advantageous than analog modulation, some of advantages are channel impairments, greater security and easier multiplexing. There are several applications of QAM modulation for domestic broadcast applications 64 QAM and 256 QAM are often used in digital cable television and cable modem applications [4]. The higher order modulation techniques are significantly less effective to noise and interference.

The practical problem in digital communication is intersymbol interference. ISI in transmitted symbols is caused by multipath fading effect. At the transmitter interference mitigation can also be implemented where by reducing the interference energy sent to co-channel users while providing the signal to the desired user [5]. Equalizers are the linear filter that provide inverse of the channel. Several channel equalization techniques are used to mitigate the ISI in the transmitted symbol. Linear channel Equalization techniques are more easy to use to combat the ISI. Most common linear equalization techniques used are ZF and MMSE equalization. Linear detection techniques significantly reduce the computational complexity on the receiver [6] with the degraded performance.

In this paper performance of linear detection techniques is analyzed for various MIMO system configurations using MQAM modulation. The performance comparison between ZF and MMSE is carried out for the $2 \times 2,4 \times 4,6 \times 6$ and $8 \times 8$ MIMO system configurations and also performance carried out for 4-QAM, 8-QAM, 16-QAM and 64-QAM between ZF and MMSE detectors, however performance improvement occurs for ZF with the increase in modulation bits/symbol.

Rest of the paper is organized as follows: Section II shows the overview of MIMO system under Rayleigh fading channel. Methodology and observations of the research are described in the section III. In section IV the simulated results are described and in section $\mathrm{V}$ conclusion of the paper is given.

\section{SYSTEM OVERVIEW}

The uncertainties in wireless channel, especially in fast fading scenarios, pose challenges in achieving the benefits in the MIMO systems [7].General block diagram of $n R x \times n T x$ MIMO system, where nTx are the number of transmitting antennas and $\mathrm{nRx}$ are the number of receiving antennas is shown in the figure. 


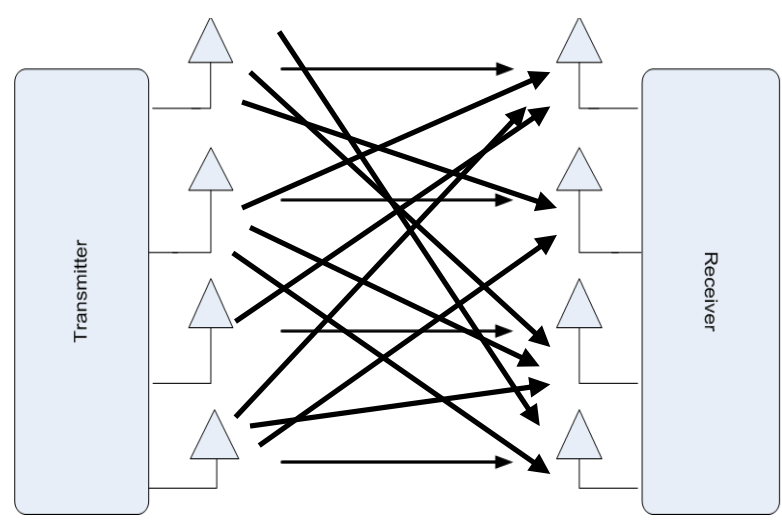

Figure 1 MIMO System Model

Considering a $4 \times 4$ MIMO system model as shown is the above figure, the above MIMO system comprised of four antennas at the transmitter and the receiver. According to the given system the data need to be grouped into the symbols of four. In the first time slot four QAM symbols x1, x2, x3, and $\mathrm{x} 4$ are transmitted from the first, second, third and the fourth antenna respectively. Thus the signal received at the receiver can be given as,

$$
\begin{aligned}
& y_{1}=h_{11} x_{1}+h_{12} x_{2}+h_{13} x_{3}+h_{14} x_{4}+v_{1} \\
& y_{2}=h_{21} x_{1}+h_{22} x_{2}+h_{23} x_{3}+h_{24} x_{4}+v_{2} \\
& y_{3}=h_{31} x_{1}+h_{32} x_{2}+h_{33} x_{3}+h_{34} x_{4}+v_{3} \\
& y_{4}=h_{41} x_{1}+h_{42} x_{2}+h_{43} x_{3}+h_{44} x_{4}+v_{4}
\end{aligned}
$$

However in the second time slot further four symbols x5, x6, $\mathrm{x} 7$ and $\mathrm{x} 8$ are transmitted from the first, second, third and the fourth antenna respectively. The channel matrix $\mathrm{H}$ is given by

$$
\left[\begin{array}{ccc}
\mathrm{h}_{1,1} & \cdots & \mathrm{h}_{1, \mathrm{nTx}} \\
\vdots & \ddots & \vdots \\
\mathrm{h}_{\mathrm{nRx}, 1} & \cdots & \mathrm{h}_{\mathrm{nRx}, \mathrm{nTx}}
\end{array}\right]
$$

Where, $h_{1,1}, h_{1,2}, h_{2,1} \ldots \ldots$ are channel coefficients, each channel coefficient is assumed to be independently identically distributed (i.i.d) complex Gaussian random variables with zero mean and unit variance. The channel assumed to be Rayleigh fading channel. The channel output for the MIMO system can be written as

$$
y=H x+v
$$

$\mathrm{y}$ is the received symbol vector $y=\left[\mathrm{y}_{1}, \mathrm{y}_{2}, \mathrm{y}_{3} \ldots \ldots . . \mathrm{y}_{n T x}\right]^{T}, \mathrm{H}$ is the complex valued Rayleigh fading channel Matrix of dimension $n R x \times n T x$, The symbol vector $\mathrm{x}$ is assumed to be uncorrelated with independent and identically distributed (i,i,d) complex Gaussian Random variables $x=\left[\mathrm{x}_{1}, \mathrm{x}_{2}, \mathrm{x}_{3} \ldots \ldots \ldots \mathrm{x}_{n T x}\right]^{T}$. The Additive White Gaussian Noise (AWGN) vectors $v=\left[\mathrm{v}_{1}, \mathrm{v}_{2}, \mathrm{v}_{3} \ldots \ldots \ldots \mathrm{v}_{n R x}\right]^{T}$ are independent and identically distributed (i,i,d) complex noise Random variables with zero mean and covariance matrix $\sigma_{n}^{2}$, where I indicates identity matrix.
The linear MIMO detection techniques related to this work are briefly discussed in below sections. Maximum Likelihood (ML) detection complexity increases with the increase in symbol size and the transmitting antennas that's why ML cannot be used in most of the practical systems. MMSE does not yield into account the correlation of the components of the converted noise due to this it is suboptimal. However ZF or MMSE detection can only achieve a diversity of order nr+nt-1 [8]. In comparison to ML receivers linear receivers are used to reduce the complexity of the system. MMSE and ZF are fast detection techniques and are easy to implement, that's the thing that motivates the major work in this paper focuses on the performance of linear MIMO receivers.

\section{OBSERVATION AND METHODOLOGY}

\subsection{Zero-forcing detection:}

Zero-forcing (ZF) is the low complexity linear detection algorithm used to estimate the transmitted signal $\mathrm{x}$ when the channel coefficients must be known at the receiver. This algorithm treats as a linear filter separating different data streams and performs independently decoding on each stream. This detection algorithm relatively shows good results at high SNR and it has very simple structure. The estimated signal using $\mathrm{ZF}$ detection is given as [9],

$$
W_{\text {ZF }}=\left(\mathrm{H}^{H} \mathrm{H}\right)^{-1} H^{H}=H^{+}
$$

Where $H^{H}$ the Hermitian of the channel matrix, Hermitian is used because the channel coefficients are complex.

The estimated transmitted symbol vector $\hat{x}_{Z F}$ of the Zero forcing detection can be written as,

$$
\hat{x}_{Z F}=W_{Z F} y
$$

Where $W_{Z F}$ is the zero forcing equalization matrix, it is equivalent to Pseudo-inverse of the channel matrix $\mathrm{H}$. The drawback of zero forcing receivers is noise amplification due to this it shows degraded performance of BER.

\subsection{MMSE Detection:}

Minimum Mean Square (MMSE) is the linear estimator that minimizes the mean square error between the transmitted symbol and the estimated symbol at the receiver [9].MMSE estimator mitigates ISI and also minimizes the noise that's why it is also known as an optimal detector. The equalization matrix for the MMSE detection is given as,

$$
W_{\text {MMSE }}=\left(\mathrm{H}^{H} \mathrm{H}+\frac{P_{n}}{P_{x}} I\right)^{-1} H
$$

Where

$W_{M M S E}$ is minimizing the mean square error $E\left(\left\|W_{\text {MMSE }} y-x\right\|^{2}\right)[10]$,

$H^{H}$ is the Hermitian of the channel matrix, Hermitian is used because the channel coefficients are complex.

$P_{n}$ and $P_{x}$ are the powers of the noise and the transmitted signal respectively. 
$I$ is the identity matrix of $n \operatorname{Tx} \times \mathrm{nRx}$ dimension.

The estimated transmitted symbol vector $\hat{x}_{Z F}$ of the Zero forcing detection can be written as,

$$
\hat{x}_{\text {MMSE }}=\left(\mathrm{H}^{H} \mathrm{H}+\frac{P_{n}}{P_{x}} I\right)^{-1} H y
$$

The above equation can be reduced as,

$$
\hat{x}_{\text {MMSE }}=\left(\mathrm{H}^{H} \mathrm{H}+\frac{1}{S N R} I\right)^{-1} H y
$$

Where SNR is the signal to noise ratio, SNR $=\frac{\text { Signalpower }\left(\mathrm{P}_{s}\right)}{\text { Noisepower }\left(\mathrm{P}_{n}\right)}$, however at high SNR the MMSE approaches to ZF detection.

\section{SIMULATION RESULTS AND ANALYSIS}

In this section the simulated results are discussed which are conducted in MATLAB for the performance evaluation of the Linear MIMO detectors such as ZF and MMSE. The parameters specification for the MIMO system model used in the simulation purposes are given in the table below. The channel used in this is Rayleigh fading channel and the channel coefficients are complex Gaussian Random variables with zero mean.

\section{TABLE I}

\begin{tabular}{|l|l|}
\hline \multicolumn{2}{|c|}{ SIMULATION PARAMETERS } \\
\hline Parameters & Specifications \\
\hline $\begin{array}{l}\text { Number of Transmitting } \\
\text { antennas } \\
\text { Number of Receiving Antennas }\end{array}$ & $2,4,6$ and 8 \\
Data Symbols & $24 \times 104$ and 8 \\
Data Frame Length & 2 to 8 \\
Channel & Rayleigh fading \\
Noise & Additive White Gaussian \\
Noise (AWGN) & MMSE, ZF \\
Algorithm & $\begin{array}{l}\text { 4-QAM, 8-QAM, 16-QAM, } \\
\text { 3igital Modulation Techniques }\end{array}$ \\
Signal to Noise Ratio (SNR) & 0 to 30 \\
\hline
\end{tabular}

The random bit sequence is generated then that is modulated with QAM modulation. The modulated signal is passed over a Rayleigh fading channel with the addition of AWGN noise. Linear MIMO detection is performed at the receiver. QAM symbols are demodulated and are converted into bits. The MIMO system performance is evaluated using Bit Error Rate (BER) vs Signal to Noise Ratio (SNR) as indicated in the fig. 2 to fig. 7 .

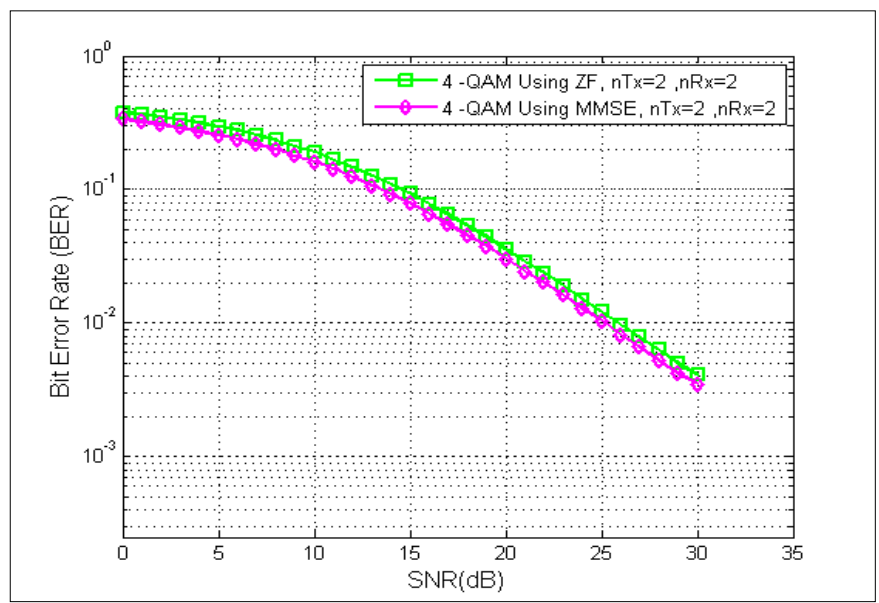

Figure 2, Performance Comparison of MMSE and ZF receivers, $n T x=n R x=2$.

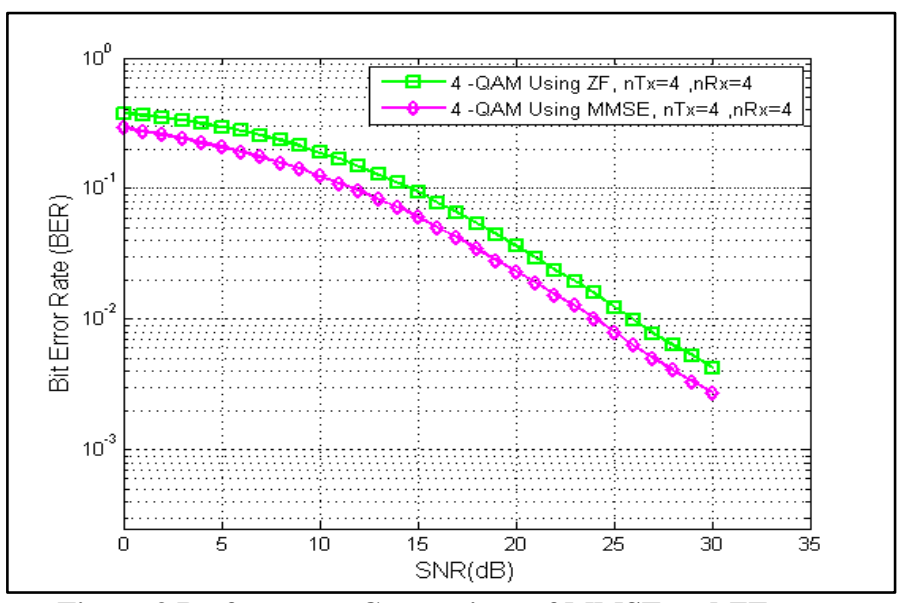

Figure 3 Performance Comparison of MMSE and ZF receivers, $n T x=n R x=4$.

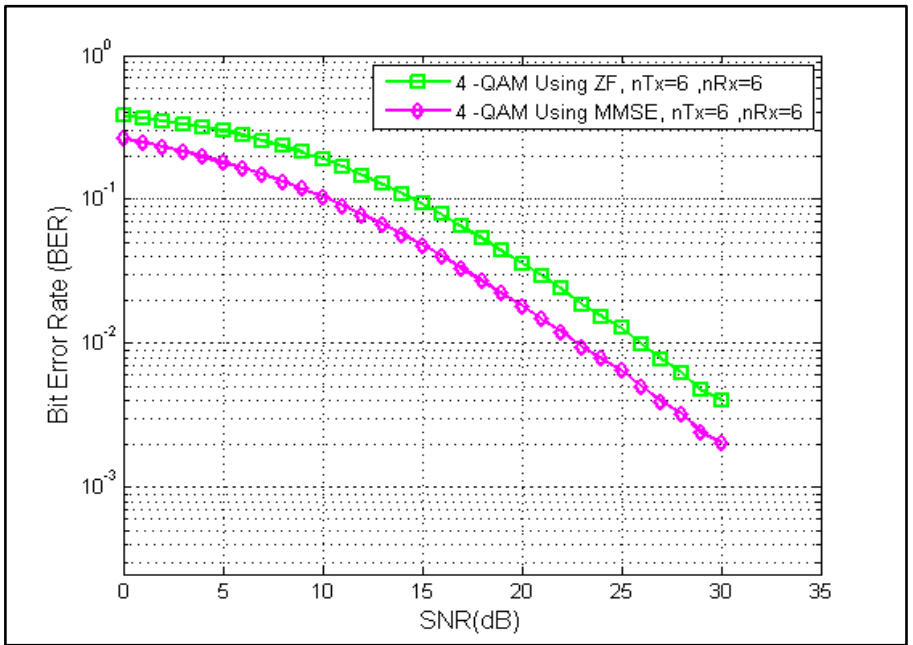

Figure 4 Performance Comparison of MMSE and ZF receivers, $n T x=n R x=6$. 


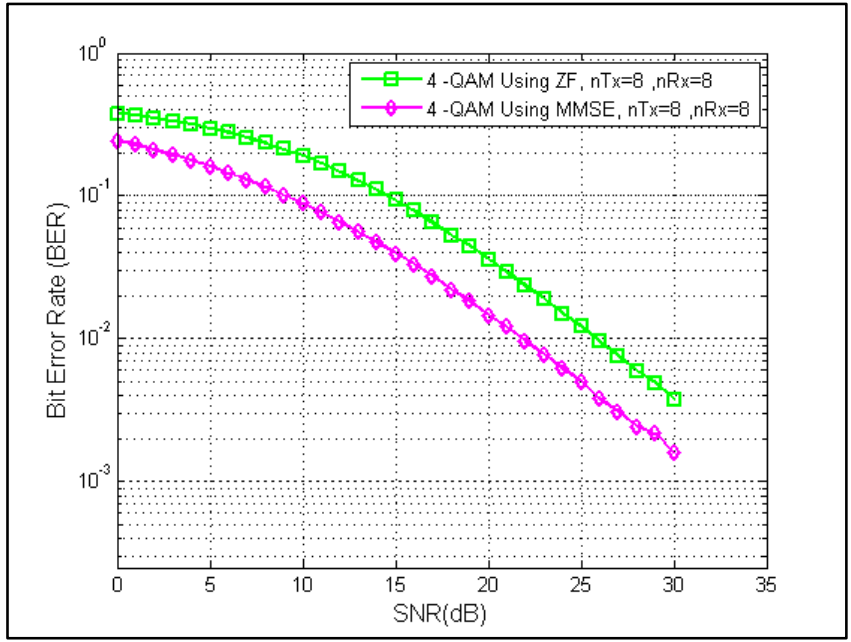

Figure 5, Performance Comparison of MMSE and ZF receivers, $\mathrm{nTx}=\mathrm{nRx}=\mathbf{8}$.

We have evaluated the performance of ZF and MMSE receivers for different MIMO system configurations. The modulation technique used in Figure 2 to figure 5 is 4-QAM modulation. It can be seen in figure 2 for MIMO system with two transmitting and receiving antennas MMSE shows $1 \mathrm{db}$ better performance than $\mathrm{ZF}$ at 10-2 BER point. It is also noted that the MMSE detector performance is close to the ZF detector. On the other hand in figure 3 at 10-2 BER point MMSE performance is $2 \mathrm{db}$ better than $\mathrm{ZF}$, while for $6 \times 6$ MIMO system configuration in figure 4 the MMSE shows $3 \mathrm{db}$ increased performance than $\mathrm{ZF}$ at the same BER point. In figure at the BER point of 10-2 MMSE shows $4 \mathrm{db}$ improved performance than the ZF.

By comparing the figure 2 to figure 5 it becomes clear that the MMSE receiver performance is better for each of the MIMO system configuration. It is also obvious that the MMSE performance is improving more by the increase in number of transmitting and receiving antennas however increasing number of antennas does not affect more on the ZF receiver. Therefore as the number of transmitting and receiving is increased in the MIMO system with the QAM modulation scheme, the performance of $\mathrm{ZF}$ and MMSE receiver also increases.

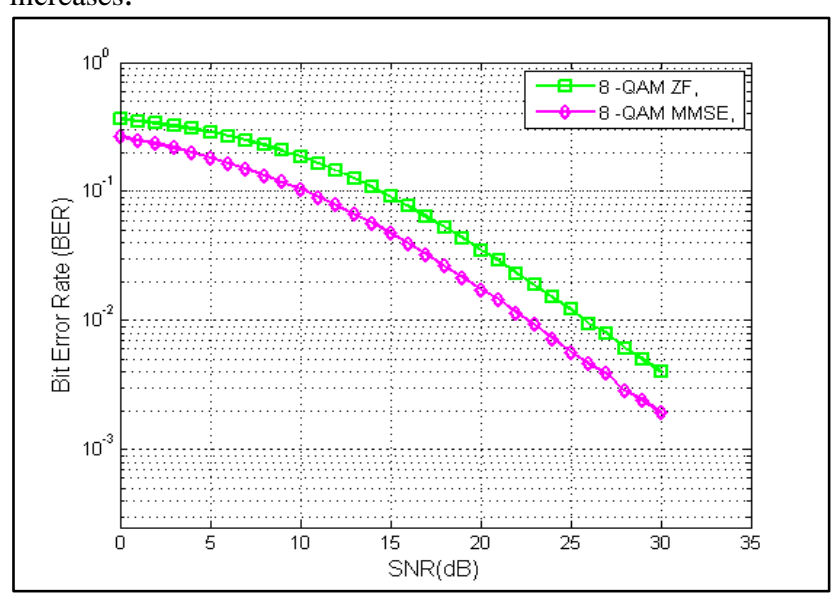

Figure 6, Performance Comparison of MMSE and ZF receivers for 8-QAM modulation.

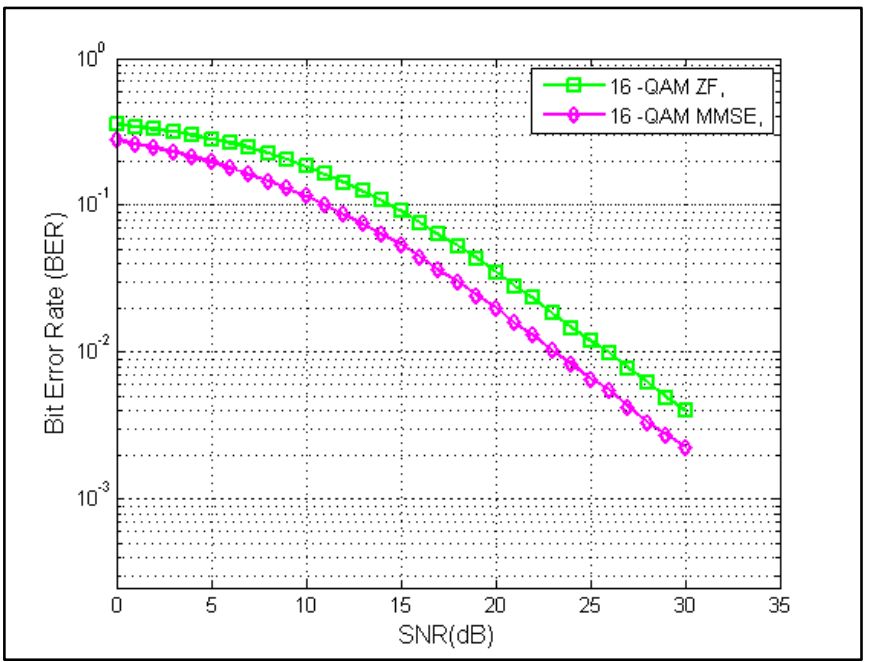

Figure 7, Performance Comparison of MMSE and ZF receivers for 16-QAM modulation.

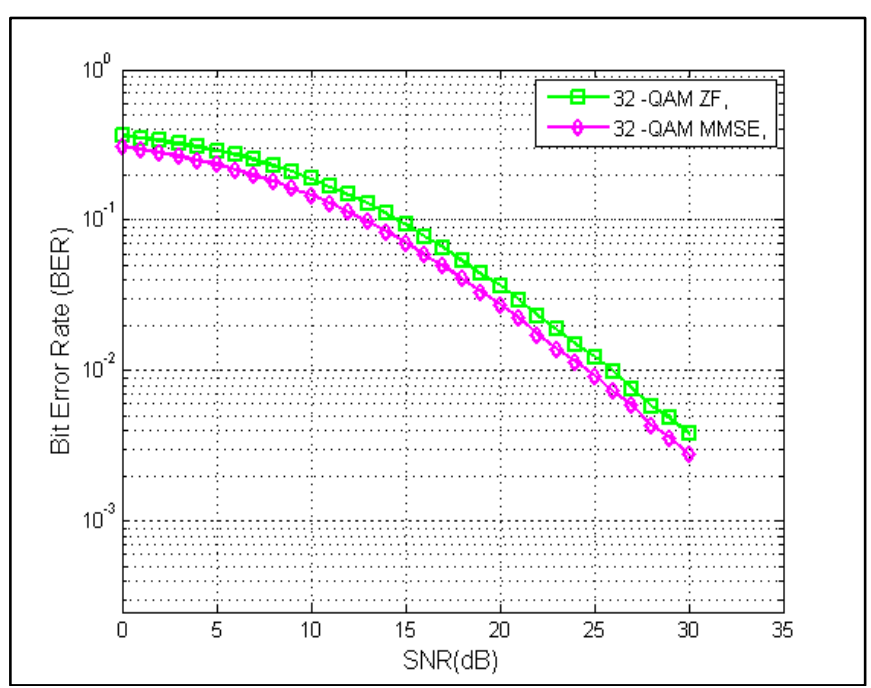

Figure 8, Performance Comparison of MMSE and ZF receivers for 32-QAM modulation.

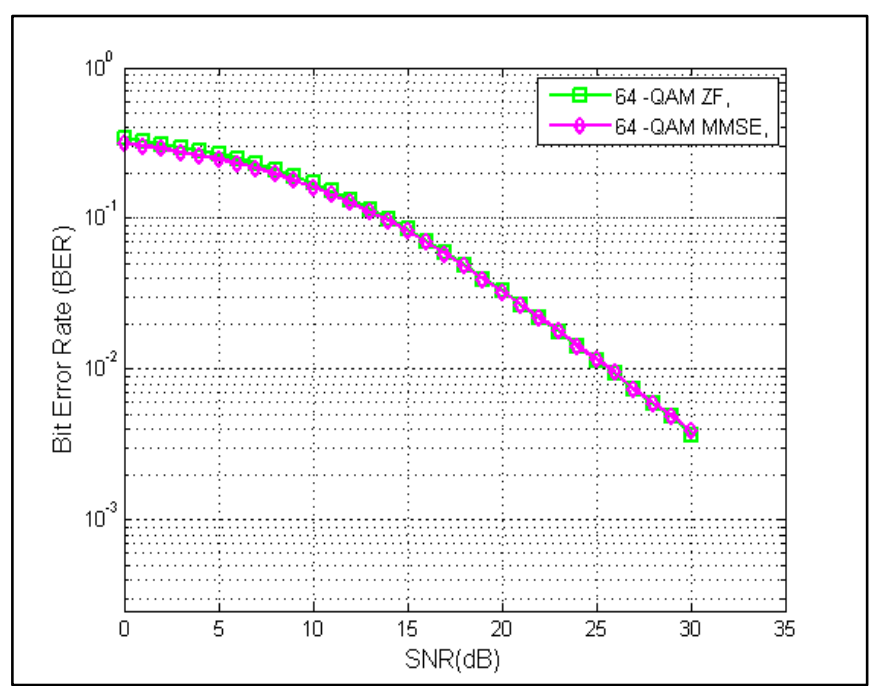

Figure 9, Performance Comparison of MMSE and ZF receivers for 64-QAM modulation. 
The results of simulations analyze performance of Linear MIMO receivers and compared on the basis of BER for the different modulation techniques such 8-QAM, 16-QAM, 32QAM and 64-QAM. In figures 5 to figure 7 the number of transmitting and receiving antennas are 4 that is the performance is analyzed for $4 \times 4$ MIMO system configuration over Rayleigh fading channel.

From the simulation results indicated in figure 6 to figure 9 it is observed that MMSE receiver shows better performance than ZF however with the increase in the constellation size the $\mathrm{ZF}$ receiver shows the same pace of curve to the MMSE receiver.

\section{CONCLUSION}

In this paper ISI in the fading channel is mitigated effectively using linear MIMO receiver algorithms. BER analysis is performed for various MIMO system configurations and for different modulation schemes as well. It is found that the performance of MMSE algorithm is better than ZF but with the increase in the constellation size both of receivers shows same performance. The reason behind is that with the increase in the constellation size the power of the transmitted signal and the data rate also increase due to this it can be concluded from equation that the BER performance of MMSE becomes weaker and approaches to ZF. In future we can use Alamouti Code and SVD Decomposition that improves the BER performance with the same system. Equalizer algorithms like MMSE can be used with V-BLAST.conclusion section is not required.

\section{ACKNOWLEDGMENT}

Authors would like express great thanks for helpful suggestions of Mr. Faizan Ali Siddiqui, Mr. Raheel Ahmed and Support from the College of Information and Communication Engineering.

\section{REFERENCES}

[1] Introduction to MIMO Systems by Rohde \& Schwarz Authored on: Sep 7, 2007 by S.Schindler.
[2] G. J. Foschini and M.J Gans, "On limits of wireless communications in a fading environment when using Mutiple antennas,"Wireless Pers. Commun., vol. 6, no. 3, pp. 311-335, Mar. 1998.

[3] V. Tarokh, N. Seshadri, and A. R. Calderbank, "Spacetime codes for high data rate wireless communication: performance criterion and code construction,", IEEE TRANSACTIONSON INFORMATION THEORY, VOL.44,NO.2, MARCH 1998.

[4] DIGITAL COMMUNICATIONS Fundamentals and Applications, Second Edition by BERNARDSKLAR, Communications Engineering Services, Tarzana, California and University of California, Los Angeles

[5] Arogyaswami j. Paulraj, dhananjay a. Gore, Rohit u. Nabar, andhelmut bölcskei" An Overview of MIMO Communication.A Key to Gigabit Wireless", proceedings of the ieee, vol. 92, no. 2, february 2004.

[6] Shih-an Yu, Student Member, IEEE, "Comparative Study of MIMO Systems with Linear Detection and Error-correction Code", Columbia University ELEN6909 Modern Digital Modulation Techniques.

[7] E. Biglieri, R. Calderbank, A. Constantinides, A. Goldsmith, A. Paulraj, and H.V. Poor. MIMO Wireless Communications. Cambridge University Press, 2007.

[8] L. Zheng and D. Tse, "Diversity and multiplexing: A fundamental tradeoff in multiple antenna channels," IEEE Trans. Inf. Theory, vol. 49, pp. 1073-1096, May 2003.

[9] Bhasker Gupta and Davinder S.Saini, "BER Performance Improvement in MIMO Systems Using Various Equalization Techniques", 2012 2nd IEEE International Conference on Parallel, Distributed and Grid Computing.

[10] S. M. Kay, Fundamentals of Statistical Signal Processing: Estimation Theory. Englewood Cliffs (NJ): Prentice Hall, 1993. 